with its vital importance. To discharge such patients before healing is complete merely because they have been long in hospital, and the serous discharge has become scanty, is surely not justifiable. If admitted at all, they must be treated with the same care until they are healed. If failure to exclude septic mischief occurs, evidenced as it will be by the supervention of hectic fever and the discharge of pus from the sinus, no reason longer exists for keeping such a patient in the hospital. His fate is sealed, no matter where or how he may be treated.*

\section{ABscess of The Hip-JoInt.}

I must also refer very briefly to abscess of the hip.joint. Here results are obtained by antiseptic treatment essentially similar to those which $I$ have described in speaking of spinal abscess. If scrupulous antiseptic dressing is maintained, along with perfect rest of the articulation, the serous discharge which alone follows the evacuation of the original contents ceases after a longer or shorter period, and if no shortening of the limb had occurred before the operation complete restoration of the normal condition may be anticipated, while in cases of partial absorption of the head of the bone incomparably better results are obtained than can be got by excision, the patient having meanwhile remained absolutely free from constitutional disturbance.

Treatment of Acute Abscess.

In ordinary acute abscesses, such as those occurring during lactation, a merely serous discharge follows antiseptic evacuation and drainage, just as in the chronic cases; but instead of the protracted course commonly followed by the latter, complete healing occurs with great rapidity, the micrococci which abound in and around. the abscess wall being speedily disposed of by the antibacteric power of the living organism, when the disturbing influence of the pent-up pus is removed and prevented from recurring, while contamination from without is precluded. In acute abscesses of a pyaemic character which one meets with occasionally in connexion with such ailments as enteric and scarlet fever, pneumonia, and the like, as well as after parturition, this typical course cannot be reckoned on, yet even in them the difference in the chances of the patients, according as they are treated antiseptically or not, can hardly be exaggerated. If strict antiseptic treatment and free drainage be adopted, and the access, therefore, of bacteria from without be prevented, the most happy results usually follow. Very soon the discharge becomes thinner, and often a mere serous exudation ultimately takes the place of the original thick pus, while the evidences of septicaemia, as indicated by high fever, rigor, delirium, and the like, pass quickly away. No washing of the cavity is desirable, provided that mischitf from without be successfully excluded. If, however, this is not accomplished, an accession of grave symptoms may supervene, and a fatal issue is a frequent result, for acute abscess of large size is often a late episode in the illness of a patient already debilitated by serious disease.

The fear which is sometimes felt of contamination of aseptic incised wounds by abscesses, of whatever nature, treated in the same ward, will be found to be absolutely groundless under antiseptic management.

\section{Conclusion.}

I have now, gentlemen, reviewe $d$ the gradually and slowly built up additions to our resources and powers in controlling the progress of wounds, and the issues of abscesses. This I have not attempted to do in full detail, but rather tried to refer to the chief principles which guide us, and the character of the materials with which we work in carrying out these principles. It is unnecessary that I should endeavour to portray the contrast which may be easily drawn between surgery as it exists to-day and surgery as I witnessed it on my introduction to hospital as a student. Let it suffice to say that we who practise surgery to day do so with a confi-

* Since these lectures were delivered reports have appeared by different surgeons of the application to surgical practice of the socal Sir Alm roth Wriatment evolved from the beautiful researches nature reforr $\mathrm{w}$ to in the toong the cases recorded are some of the in spite of the contamination of the abscess from without. efrected thus raised that such cases may in the future lose their present hepe less character : and also that the progress towards recovery uncontaminated cases treated antiseptically may be greatly accelerated. dence and a sense of safety to which the surgeons of forty-five years ago were entire strangers, and this is an outcome of the application of scientific truths. The very fact that it is such an outcome makes it as necessary now as it was declared to be at first that we shall constantly keep before our minds what those scientific truths demand of us in the treatment of each individual case. If we maintain such a mental attitude we need not strive for, or indeed expect, absolute uniformity of practice. The more clearly we recognize the principles which are the common guides of all of us, the more we shall agree in that which is essential and be free to differ as we please in carrying out the various details of our everyday procedures.

1 Lancet, July 6th, 1867. 'Ibid., March 16th, 1867. 3 Ibid., March 16th, 1867. July 6th, 1867. 2 Ibid., March 16th, 1867. ${ }^{3}$ Ibid., March p. 943. T Lister Jubilee Number of the BRITISH MEDICAL JOURNAL, Necember 13th, 1902 " 8 The A septic Treatment of Wounds, by Dr. Alfred Theodore Rake. M.B., p. 83.9 Remarks on a Case of Compound Dislocation of the Ankle with other Injuries, illustrating the Antiseptic System of Treatment, Lancet, March $19 \mathrm{th}$ and 26th, and A pril 9th, 1870. 10 Transactions of London Medical Congress, 1881, vol. ii, p. 372 11 Transactions of the Berlin Medical Congres8. 1890: also BRITISH MEDICAL JOURNAL, August 16th, 1890. 12 Dublin Journal of Medical Science, 1879, vol. ii,p. 99. 13 Erste aerztliche Hillfe, edited by Reports, vol. xxxii.

\section{DR. HUGHLINGS JACKSON'S 'VIEWS OF THE FUNCTIONS OF THE CEREBELLUM,} AS ILLUSTRATED BY RECENT RESEARCH.

Being the Hughlings Jackson Lecture for 1906.

By Sir VICTOR HORSLEY, F.R.S., F.R.C.S., Etc., SURGRON TO THE NATIONAL HOSPITAL FOR THE PARALYSED AND EPILEPTIC ; LATE SURGEON TO UNIVERSITY COLLEGE HOSPITAL.

THE Hughlings Jackson Lecture was founded in 1897, by the Neurological Society, to do honour to Dr. Hughlings Jackson, and to acknowledge the great service he has done to humanity by his work on the functions of the nervous system.

The power of genius evinces itself by the happy use of analy sis and synthesis, with as a result the discovery of principles. Dr. Hughlings Jackson's writings are all such expositions of principles, and no one rises from their perusal without realizing that his conclusions are final without dogmatism, that a subject dealt with in bis way always remains open for further scientific advancement, and finally that his reader has learnt far more than can be at nnce simply and adequately expressed. His teaching of first principles will always be gratefully remembered by the ever-increasing army of neurologists, but this is the opportunity for those of us who have enjoyed the inestimable privilege of being immediately his pupils specifically to recall the profound depth of our indebtedness to him. We feel that his precept and example has influenced us both in methods of work and in methods of thought. Of the former I need only now particularize his constant inculcation of accuracy in observation, and, above all, his insistence on the necessity for correlating disturbances of function with the structural changes found after death.

Of even greater value has been his method of thought, by which a thinker can keep his mind open to appreciate broad principles even when it is most inclined to be narrowed by close attention to detail. Above all, the intellectual width of his demonstration of the true principles of localization of function in the central nervous system, and how with the coexistence of such localization the functional activity of the nervous system as a whole can yet be estimated and philosophically considered.

Indeed, the greatest principle which underlies all he has written is the true nature of localization of function in the central nervous system-namely, that it is relative and not absolute, that conceivably every part of the body is 
represented in every nerve centre, just as it of necessity is in the single primordial ovum. And that, when the central nervous system, considered functionally, is regarded in the view of Flourens as working as a whole the determination of action by any given part is never more than re]ative.

Perhaps Dr. Jackson's view of localization in the central nervous system is not always sufficiently remembered, and more especially when we are labouring with the difficulties of endeavouring to establish a differential diagnosis are we likely to forget that the whole machine is in active operation while our attention may be drawn to one part only.

In no sense is this truer than of the difference between and yet consonance of function in the cerebrum and cerebellum. Both organs are intimately correlated and both must be considered as invariably working together. $\mathrm{Dr}$. Jackson's own confession of faith on this point is as follows :

I believe that the cerebrum represents all parts of the body, and that the cerebellum also represents* all parts of the body. ... That the cerebrum and cerebellum are engaged in any (every?) operation will, I suppose, not be doubted!-Brain, vol. $x$, p. 317 .

But it is not only the correlation between the cerebrum and cerebellum that is of importance, we have to add to these two organs a third, the equally. important spinal cord.

Dr. Jackson has for thirty years ${ }^{9}$ impressed upon us the necessity of always remembering that an interaction of these three parts is continually going on, that if one of them is put out of gear-for example, by an accidental lesion-then the functional energy of the remaining parts must assert itself. Let me here point out that this logical conclusion carries with it the further assumption that a certain degree of nerve energy is being constantly evoked, and as constant]y is passing from one part to the other, and in that sense the central nervous system does work as a whole.

In confirmation of this the experimental observations of Gotch and myself in 1891 on the marked diminution in the resting electrical difference of the spinal cord when it is separated from the brain may be quoted, ${ }^{5}$ as well as the similar diminution or fall shortly before and at systemic death. Further, the "drainage" hypothesis of inhibition recently enunciated by Professor $M$ cDougall 12 also connotes the same idea-namely, that there is a certain quan. tum of nerve energy constantly being evoked in any given central nervous system; while finally, as I have often urged, the clinical pictures of surgical shock and of traumatic neurasthenia can only be explained on the same basis.

Thus each part of the nervous system influences another, and for a long time Dr. Jackson has spoken of that share of energy emanating from the cerebrum or cerebellum (the latter more especially) as the cerebral and cerebellar influx respectively.

The evidence of its occurrence must, as he says, be a positive phenomenon, and thus the causation of (postcerebral) rigidity and tonic contracture has been regarded by Dr. Jackson under the view that if the higher centres of the cerebrum or cerebellum are constantly exerting an (efflux) influence on the lower centres, it needs only to remove the higher organ-for example, the cerebrum-to allow of the lower-for example, the cerebellum-producing effects of its own unhampered. Hence the doctrine of cerebellar influx as postulated by Dr. Jackson, but it is obvious that whatever be the ultimate explanation of early hemiplegic rigidity, his establishment of the principle of the production of motor phenomens of a chiefly tonic character by the lower level or bulbo-spinal apparatus when the highest level centres have lost their functional value, is of vital importance to neurology. His. in effect, is the converse of Setschenow's original position ${ }^{17}$ in respect of the influence of the corpora quadrigemina or optic lobes in the frog on the reflex function of the spinal cord.

The questions that rise immediately for discussion when such a principle is advanced are those which must govern its application-namely, inquiries into the specific or individual function of localized centres.

* Perhaps as the term "representation" connotes in many minds the representation of movement more especially, it may not be out of 作 of all forms of sensation.
For the present occasion of this lecture we will select the cerebellum.

To begin and end with the cerebellum, it.is partly necessary to allude to previous ideas of the action of that division of the neural axis in its relation to the cerebrum and the spinal cord. Two hypothetical views thus prevail on the interaction between the cerebrum and cerebellum.

1. The cerebellum favours the activity of the cerebrum.

2. The cerebellum hinders the activity of the cerebrum.

I do not propose to enter upon a discussion of these views; because, at the present time, they deal only with the dynamic activity of the cerebral and cerebellar centres, and their output of energy, as estimated by the force of muscular contractions, wholly without reference to the facts of reciprocal innervation, as we now understand them, thanks chiefly to Sherrington's work, ${ }^{18}$ and with very little reference to the subdivisions of the cerebellar and bulbo-mesencephalic tracts and structures.

Any systematic attempt to analyse the cerebellocerebro-spinal interaction, or to trace the paths and degrees of its influence, must be based on facts which can only be ascertained by the physiological investigation of the following tracts:

I. Cerebellum Cortico-nuclear Paths.

Cerebellar cortex to nucleus dentatus.

$$
\text { " " nuclei vestibulare }
$$

$$
\begin{array}{lll}
, & \text { nuclei vestibu } \\
, & \text { pons Varolij. }
\end{array}
$$

II. Cerebellum Nucleo-encephalic Paths.

Nucleus dentstus to nucleus ruber.

$$
\text { thalamus. }
$$

griseum centrale tecti.

"fastigii to nuclei nervi vestibularis.

III. Cerebrum Thalamo-cortical Paths.

Thalamus to "excitor-motor" area.

"equilibrial" area.

IV. Cerebellum Nucleo-spinal Paths.

Nuclei nervi vestibularis to spinal nuclei.

V. Side Paths of Correlated Importance.

Rubro-spinal tract.

I'ecto-spinal tract.

For the complete investigation of these tracts it will be ultimately necessary to trace to their original source the nerve impulses which pass along them. So far, the data to hand are very incomplete, but in order that we may, if possible, analyee Dr. Jackson's position, we can arrange the facts at our disposal, and we shall find that they justify certain positive conclusions, all of which are in gratifying harmony with his speculations.

The primary distinctions in functional or physiological value between them must be grouped as follows:

1. Cerebellar effects alone.

2. Cerebellar effects plus cerebral influence.

3. Cerebellar effects plus spinal cord influence.

Unfortunately these combinations of functions cannot be properly analysed by clinical research, and what we observe in any given patient are extremely compound effects. On the other hand, since, in accordance with the Jacksonian view of localization of function, the seat of disease in the very large majority of cases acts as the "predominant partner" among the coexcited portions of the central nervous system, the purposes of diagnosis are often sufficiently met.

Such a consideration brings us back to the point of view previously expressed, that there is reason to believe that the output or quantum of nerve energy is fairly constant for the same individual, assuming, of course, that his normal physiological health is maintained. It is, of course, an obviously necessary corollary to such a proposition, that, given a considerable output of nerve energy from one focus or centre (in this sense one organ) there must be so much less from the remainder of the nervous system.

In other words, any compound group of symptoms must, at some time, exhibit a predominant characteristic, and, if it can be detected, of necessity demonstrates the localization of the disease as before said.

Though localization can, by these means, be partly ascertained, it is through experimental investigation of the interacting encephalic organs in the lower animals alone possible to effect the differential analysis we need, for only in animals can the Galenical method of exclusion of parts be deliberately and restrictedly applied. 
So far this has been thoroughly accomplished only between the excito-motor cortex cerebri on the one hand and the lowest level bulbo-spinal centres on the other. This elemental analysis was begun by Francois-Frank and Pitres, and soon after confirmed by myself and others. Their. well.known work consisted in the demonstration that whereas the muscle-contraction phenomena evoked by excitation of the cortex cerebri were essentially clonic in character, those obtained by stimulating the pyramidal fibres of the corona radiata conducting to the lowest level centres were tonic.

Precisely this condition had been previously surmised by Dr. Hughlings Jackson as follows:

The cerebellum is the centre for continuous movements, and the cerebrum for changing movements. Borrowing medical terms to describe normal physiological conditions, the former (that is, cerebellar) are tonic, the latter (that is, cerebral) clonic.*-The Mredical Examiner, April 5th, 1877.

In all diecussions by Dr. Jackson of the "cerebellar" influence as contrasted to "cerebral" influence, his conclusions being drawn from clinical evidence, have been arrived at in full cognizance of the fact that all the groups of lower centres and paths, that is, cerebellar cortex, cerebellar nuclei intrinsic and extrinsic, as well as the bulbo-spinal system of lowest level centres are in action and co.operation.

The hind-brain, in short, from the point of view of physiological topography, consists wholly of Dr. Jackson's lowest levelt of representation, to which the cerebellum, forming an appendage or "anhang" to the direct system, is superadded. It is probably owing to the development in the higher mammalis of the specialized pyramidal projection system that the cortex cerebri has physiologically become so different from the numerous lower nerve centres in the mode of its discharge.

In saying this, however, it must not be forgotten that even this difference is not absolute, as indeed every instance of ankle clonus suggests, and Schäfer and $J$ proved experimentally in 1885 that the outflow of every nerve centre is fundamentally a combination of tonus and clonus.

Precisely as with the representation of movement, so is the character of the outflow different according to the proportionate degree of one or other of these two features of tonus and clonus. Hence Dr. Jackson's assertion of the essentially tonic characteristics of the discharge of the lowest level centres is not only of vital importance to cerebellar physiology, but it is in accord with the principles which underlie all nerve activity.

The best illustration that can be offered of the absolute truth of his general position on this point is that derived from a study of the epileptic convulsion. For this we happily. need only to compare middle level and lowest level fits. In 1885-7. I found that in fits obtained by Magnan's absinthe method the type was clonic or not according to the proportionate activity of the cerebral cortex. Boyce, at my suggestion, removed a cerebral hemisphere in cats, and proved that such extirpation of the highest cerebral influences resulted in fits of a strikingly tonic character. I proposed this year to $\mathrm{Dr}$. Bouché, who has for some time been investigating at University College the details of the epileptic convulsion, that he should repeat these experiments to settle finally the question, and this he has done very completely. A good example is the following experiment which I witnessed with him. A cat, three weeks after removal of the left cerebral hemisphere, in excellent condition (that is, to superficial appearance normal, walking, etc., but exhibiting the typical atopognosis, mouvement de manège, etc., of an animal deprived of one cerebral hemisphere), was etherized, and the right (the remaining) hemisphere found to be normally excitable to electrical stimulation. A small dose of absinthe was injected into the jugular vein, and the typical result obtained of a tonicclonic fit (chiefly flexor) on the left side of the body, but only a tonic (chiefly extensor) fit on the right. The attitude of the trunk and head was that of moderate flexion: While such a fit was in active development we made an instantaneous section of the mesencephalon. Immediately the clonic movements were changed for tonic

* The italics in brackets are mine $-\mathrm{V}$. $\mathrm{H}$

. + In Dr. Jackson's own words: "The lowest level extends, it is singgested, from the tuber cinereum to. the conus medullaris. It is inade up of sepsory and motor centres of the cord, medulla, pons and

5 in the whole body, the head was powerfully drawn backwards, the left limbs and trunk gradually sharing in the extensor movement.

This sudden conversion from (what remained of) the higher to the lower type of fit was very impressive, and constituted a remarkable demonstration of Dr. Jackson's dictum, that "Speaking very roughly and neglecting some parts of the body, the cerebellum represents movements of the skeletal muscles in the order trunk, leg, arm, preponderatingly extensorwise: the cerebrum represents movements of the same muscles in the order, arm, leg, trunk, preponderatingly flexorwise." 'Dr. Bouché has established the constancy of this phenomenon by a series of successful ablations of one cerebral hemisphere and subsequent exploration with absinthe.

Perhaps the most brilliant experimental proof of Dr. Jackson's position is Leonard Hill's series of experiments ${ }^{7}$ in which he varied at will the clonic or tonic character of an absinthe fit by respectively leaving open or clamping the arterial supply to the cerebrum.

So, too, as regards the lowest level of all-namely, the spinal centres. The phenomenon first discovered by Schiff, ${ }^{16}$ and confirmed by myself and other workers, of extensor convulsions of the tail and hind limbs occurring even after section of the spinal cord, is a clear illustration of the tonic-extensor character of a lowest level fit.

Finally, Loewenthal, in an interesting work ${ }^{10}$ on the effect of anaemia on the spinal cord, obtained spinal fits in which, with flexion of the spine and hips there occurred extension of the tail, knees, and ankles.

An extreme example of such tonic response attributable in the main to cerebellar influence is illustrated in Dr. Jackson's communications published in 1871. The extreme contracture extension of the head (with lateral attitude in Figs. 1 and 2) and lower limbs and tonic flexion of the fore limbs, are clearly demonstrated in the sketches by Sir Stephen Mackenzie, whose absence tonight we all regret. But we must postpone for a few moments the determination of the precise source of the representation of these movements.

With the guidance of Dr. Jackson's principles of localizacion we must now endeavour to trace out the nerve impulses which have this power of evoking such a definite and specific association of muscular phenomena.

After what has been stated it follows that the direct investigation of the problem of Dr. Jackson's views on "cerebellar influx" naturally begins with the cerebellum itself.

As Thomas was probably the first (on anatomical grounds) to suggest, ${ }^{20}$ the cortex cerebelli and the (intrinsic) nuclei cerebelli must now be considered as two distinct organs. Dr. R. H. Clarke and myself, ${ }^{3}$ by means of lesions actually restricted to the cortex cerebelli and tested by the Marchi method, have proved this opinion to be a neurological fact, and have demonstrated that:

1. The cortex cerebelli sends no direct axones via the cerebellar peduncles to the brain or spinal cord.

2. The cortical efferent axones terminate in the intrinsic nucleus dentatus, nucleus fastigii and nucleus emboliformis vel globosus.

3. The intrinsic nuclei send efferent axones to the cerebral, spinal, and paracerebellar-that is, bulbarnuclei.

The cytological investigations of Kölliker, Ramon y Cajal, etc., Retzius by the Golgi method, and Clarke and myself with polychrome blue, of these nerve centres, confirm the results obtained by the degeneration method, for whereas the cerebellar cortex is, histologically speaking, a structure per se,* the intrinsic nuclei are morphologically homologous with the efferent apparatus of the bulbo-spinal system. Particularly is this the case with the roof nucleus, one class of cells therein being typically spinal efferent in character.

So much for morphological evidence respecting the nature of the parts of the cerebellar apparatus. A phenomenon like that of tonic muscle contraction and the source of its maintenance, however, can only be investigated physiologically.

In $1904 \mathrm{I}$ made a few experiments on dogs at Dr. Jackson's suggestion as follows :-In one group the mesen. cephalon was divided at the level of the intercollicular

* The nearest morphological homologue to the Purkinje cell system is Clarke's column in the spinal cord and the cells of the "cerebra]. root of the fifth merve. 
groove. The degree of the resulting decerebrate rigiditythat is, Dr. Jackson's " cerebellar influx" was then tested during and after removal of the cerebellar cortex in sections from above down. The hypertonus was not appreciably affected by such extirpations until the section involved the intrinsic and paracerebellar nuclei.*

In another group of bat two experiments a large horizontal lesion separating the dorsal half of the cerebellar cortex, omitting the nuclei (save in one case where one of the nuclei fastigii was destroyed), was made three weeks before the division of the mesencephalon. In these cases, also, no appreciable difference was observable in the decerebrate rigidity from that seen in normal animals.

Finally, this is the place to refer to the work accom plished by Dr. Thiele ${ }^{19}$ in his investigation of the sources of the phenomena of hypertonus known since Sherrington's original investigation as decerebrate rigidity. Thiele found that, apart from the autonomic action of the spinal cord, the plane of the principal source of the motor impulses evoking the rigidity is the level of the paracerebellar nuclei (that is, Dieter's nucleus, nuclei vestibularis, nucleus magno-cellularis substantiae recticularis, etc.).

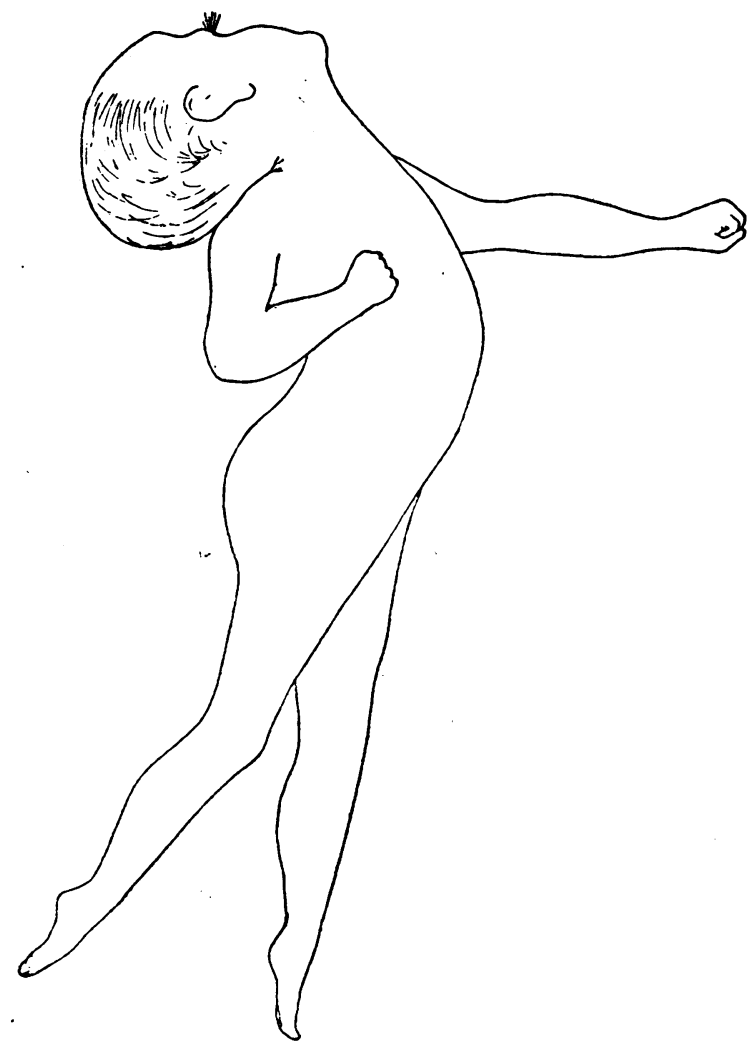

Fig. 1.-Tetanus-like seizure.

(Case of tumour of the middle lobe of the cerebellum.)

So far, then, the evidence by experiments on disordered function tended to show that the immediate source of the "cerebellar influx" must be looked for in the basal or nuclear region of the cerebellar complex, and that the activity of the cortex cerebelli, though physiologically antecedent thereto, is not essential to the maintenance of the contracture phenomenon. The next step, naturally, was the direct re-exploration of the cerebellum, and that Dr. Clarke and myself have entered upon.

During nearly one hundred years various investigators have endeavoured to obtain evidence of cerebellar function by the excitation method, $†$ either chemical, mechanical, or electrical, but, as a critical survey of the methods employed and of the results we have obtained up to the present show, it is probable that the effects observed by our predecessors were due to irritation of the subjacent nuclei and neighbouring tracts rather than to excitation of the

* In this connexion it is important to remember that Schiff always contended that the earlier results of Flourens were correct, and that in the production of cerebellar phenomena of disturbance $n$ effect was obtained until the lesions involved the "midde" of the ritho

method of investigation was, of course, in use for centuries before the excitation method. cortex, except when the intensity of the stimulus was very high-that is, when the whole apparatus of cortex and nuclei is simultaneously thrown into an active state.

The differential exploration of the cerebellum entails among many other problems, first, that of focally irritating any given point (square or cubic millimetre is the unit adopted by Clarke and myself); and, secondly, of marking and destroying it for the purpose of accurate localization, and of tracing secondary degenerations therefrom.

We* have, as described in a preliminary communication, been enabled to do this with a special apparatus carrying, by glass insulated fine needles, a current which.can be readily changed from faradic to constant at will, to any desired spot in the encephalon.

This method of investigation has brought us to the following conclusions :

First Conclusion.-That the cortex cerebelli is inexcitable when compared to the cortex cerebri of the gyrus centralis anterior (ascending frontal gyrus).

The immediate inference from this result is that the cortex cerehelli is not to be regarded as a motor centre or series of such centres, but as an afferent receptive centre

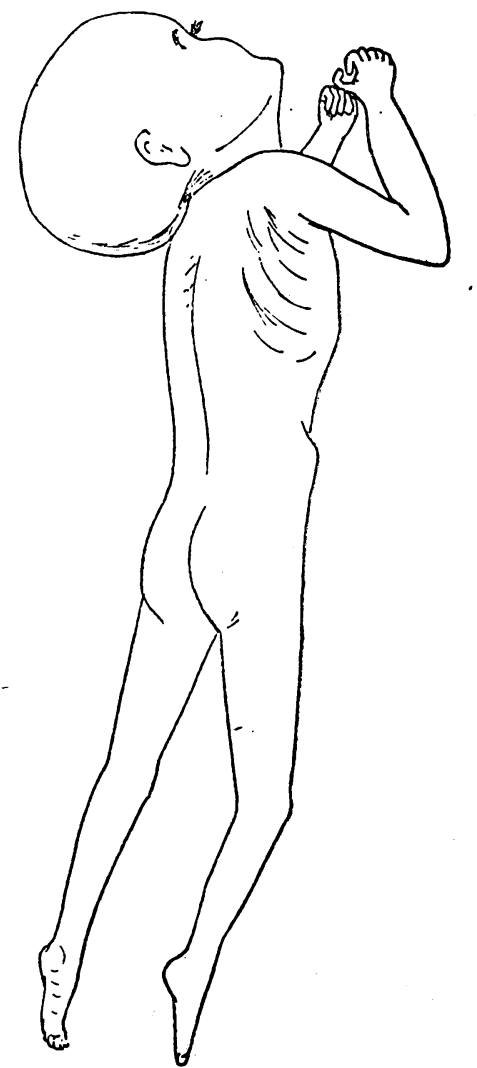

Fig 2-Cerebellar paralysis with persisting rigidity.

Fig. 2.-Cerebellar paralysis with persisting rigidity.
"Cerebeliar attitude" in a case of tumour of the middle lobe of the cerebellum.)

from which axones pass to the ventrally-placed efferent nuclei. In fact, the cortex cerebelli is somewhat parallel in arrangement to the pallial visual cerebral centre.

It must now be shown how far this position has been already established by previous researches on other lines and methods.

The vermis region of the cortex cerebelli is the end station of the dorsal spino-cerebellar tract (of Flechsig) and the ventral spino-cerebellar tract (of Gowers). These paths are sensory tracts of the second order in the central. neuronic sygtem, and therefore are really homologous to the fillet. In that case the cerebellar cortex in the general scheme of the nervous system cannot be considered physiologically higher than the ventro-lateral region of the thalamus.

The termination of these spinal tracts of fibres in the cortex cerebelli discovered by Mott ${ }^{13}$ deserves, but bas not yet received, a minute numerical analyis. Mott traced the dorsal spino-cerebellar tract to the middle lobe

* Henceforth in the lecture, wherever the expressions "we." "us "our" are used, they must be understood to refer to Dr. Clarke and myself. 
(Elliott Smith) of the vermis and the ventro-lateral tract to the lobus centralie, while the further development of the nature and functions of these paths we owe to Tooth Cohnstamm, Auerbach, and Sherrington.

I have myself made in different animals minutely limited lesions of both tracts, and like all other observers have fully confirmed Mott's original description. The direct cerebellar tracts have of recent years been made the subject of closer physiological study. Thus Marburg found that section of the tractus spino-cerebellaris dorsalis (Flechsigii) was followed by extension of the homolateral limbs which were abducted from the trunk and in a state of hypotonia although sensation and the spinal reflexes were both normal. So, too, Bing.

The time is not yet ripe to take advantage of the careful clinical work of Petrén on the ventral tract, but the general conclusions which can be justifiably drawn from the facts at our disposal, confirm Dr. Jackson's position that the atactic helplessness is primarily due to loss of information - that is, of afferent impulses.

As regards the question of the posterior columns being through their terminal bulbar nuclei partly a spinocerebellar tract, it ought, perhaps, to be added here that, like many observers, I have not traced any fibres into the inferior peduncle from the posterior columns.

This last-named anatomical fact is but an extension of the physiological position arrived at by Professor Hermann Munk, who demonstrated that section of the posterior columns did not produce ataxy whereas lesions of the posterior roots did.

Time unfortunately does not permit me to discuss further the enormously interesting and important work of Hering, ${ }^{6}$ Mott and Sherrington, ${ }^{14}$ Trendelenburg, ${ }^{21}$ and especially of Bickel, ${ }^{1}$ in this part-namely, the sensory or afferent side of the subject. I must strictly confine myself to the " motor" effects.

We recognize as the result of these morphological* and physiological considerations, that the cortex cerebelli is an afferent recipient organ, as Edinger has always taught, and that the impressions from the trunk and limbs which it receives by these secondary neuronic systems from the spinal segments of the cord are of a special character being already co-ordinated. What is true of the spinal afferent paths is apparently also true of the cranial nerve tracts, except that the intrinsic cerebellar and paracerebellar nuclei additionally receive primary collaterals directly from the entering root fibres.

To return now to direct physiological investigation of the efferent phenomena. Clarke and myself have found that while excitation of the surface of the cerebellum is without effect, when on the contrary the insulated needles penetrate the cerebellar cortex, a minimal stimulus gradually becomes maximal as the needle points are made to approach the intrinsic nuclei, which are highly ex citable, and give rise to such precise motor effects as to establish as follows our second conclusion.

Second Conclusion.-That the intrinsic and the paracerebellar nuclei are the efferent mechanisms of the cerebellum.

To use Dr. Hughlings Jackson's terminology, these nuclei are the immediate source of the " cerebellar influx," the cortex cerebelli standing in the same causal relationship to the resultant motor effects as does the afferent side of every nerve centre.

The muscular contractions which follow excitation of these nuclei must be surveyed from two points of view to compare them with Dr. Jackson's opinions; first, the topography of the part of the body represented, and secondly, the character of the muscular contraction observed.

\section{(1) Topographical Representation in the Cerebellar and} Paracerebellar Nuclei.

As yet our researches are not completed, but provisionally it may be asserted that the essential motor representations of the parts of the body in the intrinsic and paracerebellar nuclei are in the former the movements of the

* Previous to the determination of the existence of direct spinocerebellar afferent paths, the existence of a relationship of the spinal ally suggested the middle lobe of the cerebellum had been theoretic$1864, p .340$ ). from consideration of the relative evolution of : (1) tactue sensibility of the skin of the body-that is, spinal distribution-an of the lateral cerebelfar lobes; of (2) muscular power and co-ordinated " muscular sense associated with development of the middle cerebellar lobe:" I have to thank Dr. Hughlings Jackson for kindly
referring me to Prideaux's paper. eyes and head, in the latter more especially those of the trunk and limbs.

(2) Character of the Muscular Contraction.

The character of the motor $t$ ffects obtained from excitation of the efferent side of the cerebellar apparatus is very striking and precisely of the kind originally ascribed by Dr. Jackson to it, namely, tonus and hypertonus. The movement even of the eyeballs is a steady swing over, which increases in contractile force as the excitation continues and without any clonic intermission of the motion or any development of any after-effect when the stimulation ceases. This being understood, the next questions respecting the character of movements derived from the efferent cerebellar mechanism are: $(a)$ their relation to the middle plane of the body; $(b)$ differentiation between flexion and extension.

(a) Relation of the Cerebellar Infux to the Mid-plane.We find that when the stimulus is unilaterally applied to the intrinsic cerebellar nuclei, the effect as gauged by the action of the systemic muscles is homolateral, contrasting with the typical cerebral contralateral phenomens. A demonstrative example and proof of this principle, which was first enunciated by Luciani, ${ }^{1 i}$ and especially by Risien Russell, ${ }^{15}$ is afforded by the result of our direct excitation of the upper part of the dentate nucleus alone, which we find evokes deviation of the eye of the same side, followed by conjugate deviation of both the eyes and head from the middle line to the same side.

So, too, excitation of the basal region of the dentate nucleus and the upper divisions of the paracerebellar nuclei of one side evokes powerful bicipital flexion of the homolateral elbow.

(b) Relation of Flexion to Extension.-As regards the second point, Clarke and myself find, that while excitation of the basal region of the dentate nucleus with a maximal stimulus will produce, in addition to the homolateral conjugate deviation of the eyes and head, flexion of the homolateral elbow, a deeper excitation, that is, of the paracerebellar nuclear region itself, produces widespread and additional effects, namely, extension of the contralateral elbow, hyperextension of the neck and trunk, with powerful extension* of the lower limbs. While, therefore, the forelimb is flexed at the elbow the hind limb is extended with the trunk.

Before pointing out the identity of these movements, we have observed, with those Dr. Jackson has taught for thirty-five years to be characteristic of the cerebellar apparatus and lowest level centres, I must revert for a moment to the debatable question of special represents tion in the central nervous system of the progression-like movement of extension of one limb associated with flexion of the corresponding contralateral limb, which is involved in the discussion of our observations on the paracerebellar mechanisms.

The analysis of such regular and adjusted alternation of motor function carries with it several fundamental considerations, of which the most important is the first, namely, what is the highest plane in the neural axis from which such a characteristic phenomenon can be elicited by direct excitation?

Sherrington had in his work on decerebrate rigidity proved the existence of a co-ordinated organization of the lowest (spinal) centres for the simultaneous performance of similar movements by alternated and contralateral limbs, and had proved the alternated character of representation in the spinal centres. Thiele then, in an exhaustive inquiry by the excitation method, combined with previons exclusion of the pyramidal tracts by degeneration, demonstrated that in the posterior third of the optic thalamus there exists a "centre" for progression -that is, walking.

The ablation experiments of various observers, especially Goltz, had proved for many years that, roughly speaking, the cerebral pallium was, in the quadrupedal animals at any rate, not absolutely essential to the functions of standing and walking.

It follows, therefore, that the compound movement of walking is at least represented in the red nuclear region, and that one efferent path is therefore Monakow's or * For a perfect illustration of this cerebellar movement in man, see A, Fig. 1, of Dr. Jackson's paper, published in 1871. While such extension is observed to be the end result it is necessary to state that the hamstring and sural museles are also in action, through with Jackson's words. 
the rubro-spinal bundle. Progression is, of course, an associated movement of primal importance and of coordination. It is therefore reasonable that it should have $a$ parallel representation in the paracerebellar nuclel, just as in the thalamus, and finally in the elementary manner of the reciprocal association of flexion and extension in the spinal segments.

We therefore consider the representation of the limbs in the paracerebellar nuclei, as far as the upper limbs are concerned, to be compound and partly of the progressionmovement type. But it must be further noted that in progression. (for which the attitude of standing is practically requisite) the action of the remainder of the body, namely, the trunk and lower limbs, is especially that of extension. Thus the trunk is either held straight in extension or over-extended so as to cause a dorsal concavity, while the lower limbs are extended as aforesaid; hence in man and the erect apes standing is impossible after destruction of the paracerebellar mechanisms.

The "cerebellar influx" of the trunk and lower limbs is, therefore, par excellence extension, but for the minuter and proportionate representation of flexion and extension in the cerebellar and paracerebellar nuclei reference must be made to a forthcoming paper by Dr. Clarke and myself.

Enough has now been said to show that Dr. Jackson's views of the nature of the cerebellar motor output, and his mode of interpreting the stimulation effects of gross cerebellar lesions, have been amply substantiated by the findings of experimental differentiation, but his facts of illustrative interest must now be again quoted. The republication of his description of two cases on which his views have been in part founded makes this easy.*

It will be recognized that Dr. Jackson's conclusions were founded on instances of massive bilateral excitation of the whole cerebellar complex, and therefore in his cases and Sir Stephen Mackenzie's striking sketches, we see that the features of the alternate movements of progression are entirely overwhelmed by the cerebellar disturbance with as resultant a strikingly peculiar position of the body, namely, hyperextension of the head, trunk and hind limbs associated with powerful flexion of both elbows.

I think it must be granted that, under the circumstance of secondary intraventricular distension occurring in such cases, the removal of the cerebral influx is a natural consequence of the condition. This special combination of muscular contractions, which Dr. Jackson has termed " the complementary inverse of epileptiform seizures," is comparatively rare, but as a contribution to the subject may allude to an instance of glioma cerebelli in a boy whose case I recently reported to the Society. In that case we repeatedly observed severe "cerebellar fits" of Dr. Jackson's type, and an operation enabled a glioma of considerable size to be removed from the right lateral lobe of the cerebellum. The boy remains in perfect health, and it can only be assumed that the paracerebellar structure were at least severely pressed upon only and not destroyed. So, too, in Dr. Farquhar Buzzard's very interesting case of diagnosed lesion of the cerebellum, a like affection of the nuclei doubtless existed. The discussion of the subject of extension, as Dr. Jackson's characteristic muscular movement, resulting from cerebellar and lower level centres' active influence cannot be closed without allusion to his teaching concerning the representation of eye movements in the cerebellum. From the first Dr. Jackson has considered that since the cerebellum is the essential organ in locomotion and progression, as just narrated there must be represented therein associated ocular movements.

Now, in accorjance with this view, Dr. Clarke and I have lound that the eye movements described by Ferrier as obtainable by excitation from the surface of the cerebellum; owe their motor genesis, not to locslized centres in the cortex cerebelli, but to foci in the intrinsic nuclei.

We have found that the simplest movement in a bin. ocular vision animal like the monkey is derived from the dentate nucleus, and consists of a conjugate deviation to the homolateral side, and further, that as the excitation point approaches the midline (and the neighbourhood of the nucleus of the sixth nerve below), differences in deviation tend to appear.
The analysis of these functions will take some time probably years, to complete, but one condition is well worthy of notice, namely, that originally observed by Magendie, which Dr. Jackson has spoken of as skew deviation, and in which the eyes are directed in opposite ways. Thus, one visual axis is directed up and in, while the other is down and out.

Dr. Jackson has always urged that this apparently parodoxical phenomenon is really only half a normal binocular conjugate deviation, and he traces skew deviation to a destructive lesion affecting one half of an individual's apparatus for the locomotory appreciation of distance and position in space, the localization of such an apparatus being as yet incomplete, but unquestionably in the temporo-cerebellar complex. The experimental illustration of the truth of this view occurred by the following happy accident.

Having for other purposes selected the armadillo (Dasypus villosus) as almost the lowest available mammalian type of cerebellum, Dr. Clarke and I were gratified to find on excitation of the intrinsic nuclei that skew deviation was the rule in this animal, ${ }^{*}$ and that inasmuch as it is remarkably monocular in almost every position of its head, it clearly illustrates Dr. Jackson's views most completely, since it normally employs but one half of a binocular apparatus.

The coaptation of eje movements with those of the limbs in locomotion of the body by means of the limbs is, theoretically, a fundamental physiological fact in man and the higher animals. Dr. Clarke and I suggest, therefore, that the true results of cerebellar excitation are those which give first place to the representation movements of the eye rather than of the limbs, which conclusion is in general accord with Ferrier's pioneering observations.

The antecedence of eye movements in all skeletal mus cular actions is well recognized in most cases, but the fundamental importance of such time relation in cerebellar physiology has scarcely been so much appreciated.

\section{Conclusion.}

This brings me to the general conclusion with which any statements of cerebellar function must now be summarized-namely, that all research, ancient and modern, tends to confirm the view of Flourens, Luciani, Jackson, and Edinger, that the cerebellar cortex is the first chief station of representation of the afferent basis of movements of all the skeletal muscles.

Gratefully to acknowledge to Dr. Jackson his share in bringing order out of confusion in this and many other departments of neurology is the object of our meeting here; and greatly as I feel the honour of being selected as the Society's representative to-day, I esteem the charge of communicating your thanks to him as my highest privilege.

May I add the expression of our hope that we may long continue to enjoy his, illuminating speech and his stimulating example?

LITERATURE.
(The following titles of communications are only quoted for certain special

Uner see Untersuchungen über den Mechan¿smus der nervösen Bewegungsregulation, 1903, Encke, Stuttgart.

3 Clarke and Horsley: Brain. 1905, vol. Xxviii, p. i3. Annual Meeting, Clarke and Horsley: Brain. 1905, vol. Xxviii, p. 13. Annual

Britsh Medical Asociation, BRITISH MEDICAL JOURNAL, 1906.

5 Gotch and Horsley : Phil. Trans. Roy. Soc., 1891, vol. clxxxil, p. 267. petale Ataxie," Neurologisches Centralblatt, 1897, Bd. 16, p. 1077.

7 Hill : Phil Trans, Roy, Soc, 1900, vol exciii, p. 106.

8 Horsley: "The Cerebellum. The Boyle Lecture for 1905." Bale, London. "Address in Surgery," BRITISH MEdiCAL JoURNAL, 1906. 9 Hughlings Jackson: For collected literature by Dr. Jackson see Brain, 1903, vol. XXvi, p. 356. "The First Jackson Lecture," BRITISH MEDICAL JOURNAI, 1898, vol. i, p. 64

Loewenthal : Brain, 1902, vol. Xxv, p. 274

Luciani: Il Cervelletto, 1891.

, 1903 , vol. Xxvi. p. 153

13 Mott:-Brain. Monatschrift für Psychiatrie und Neurologie, 1897, Bd. 1, S. 104:

15 Mott and Sherrington: Proc. Roy. Soc., 1895, vol. lvii, p. 481

15 Risien Russell : Phil. Trans. Roy. Soc., 1894, 1896. Journal of

16 Schify: 1894, vol. Xvil, p. 1 .

(18chiv fur die gesammte Physiologie, 1883, Bd. xxx, s. 202.

18 gherringow : Archiv fir die gesamm!e Physinlogie, 1875, Bd. 10 . 18 gherrington:

ogy, 1900, vol. ii.

Thiole: Journal of Physiology, 1905.

Thomas : Le Cervelet, 1897.

Anatomie und Physiologie. Physiologịche

* The three anima!s, monkey, dor, armadillc, thus arranged, show very strikingly the increase in skew deviation as the vision becomes very strikingly
less binocular. 